\title{
Perceptrón memoria de correlación multicapa para predecir la violencia sistemática contra la mujer por su pareja en la Sierra del Perú
}

\section{Multilayer Perceptron correlation memory to predict systematic violence against woman by their partner in the Sierra of Peru}

\author{
María Estela Ponce Aruneri y Erwin Kraenau Espinal \\ Universidad Nacional Mayor de San Marcos, Facultad de Ciencias Matemáticas. Lima 01
}

DOI: https://doi.org/10.33017/RevECIPeru2013.0008/

\section{Resumen}

En este trabajo se muestra que el modelo perceptrón memoria de correlación multicapa permite predecir el tipo de violencia que sufren las mujeres mayores de 14 años unidas o alguna vez unidas, residentes habituales en las viviendas circundantes de los Centros de Emergencia Mujer ubicados en la sierra del Perú; así como asociaciones importantes entre algunos estilos de crianza, relaciones familiares y percepciones acerca de los roles de género, con el tipo de violencia que ejerce el esposo/ compañero. Utilizamos 1126 casos de la base de datos proporcionada por el Ministerio de la Mujer y Poblaciones Vulnerables (MIMP); para los que se construyeron catorce variables de entrada, una que se refieren a estilo de crianza recibido, tres a relaciones familiares, nueve sobre percepciones acerca de los roles de género y una referida al ámbito geográfico; siete variables de salida que se refieren al tipo de violencia contra la mujer por parte de su pareja o ex -pareja. Para la etapa de diseño del modelo perceptrón memoria de correlación y entrenamiento de la red aplicamos el algoritmo de retropropagación (backpropagation), la función de activación utilizada para este caso fue sigmoidal (logística), calculamos el error cuadrático para evaluar el error cometido por la red en cada patrón(observación); y finalmente regla delta generalizada para modificar pesos y umbrales. Se elaboraron dos programas en Matlab, para aplicar el modelo perceptrón memoria de correlación multicapa con una y dos capas ocultas. El modelo perceptrón memoria de correlación multicapa, con dos capas ocultas proporciono mejores resultados, $80.34 \%$ de predicciones correctas; y muestra que la violencia familiar y la percepción acerca del rol de género son factores importantes en la predicción del tipo de violencia que padece la mujer unida o alguna vez unida por su pareja, y que reside en la sierra del Perú.

Descriptores: Memorias de Correlación, perceptrón multicapa, predicciones, violencia contra la mujer.

\begin{abstract}
In this research shows that the correlation model memory multilayer perceptron to predict the type of violence experienced by women over 14 have ever joined or united, ordinarily resident in the houses surrounding the Women's Emergency Centers located in the sierra del Peru, as well as significant associations between certain parenting styles, family relationships and perceptions about gender roles, with the kind of violence exercised by the husband / partner. We use 1126 cases of women from the database provided by the Ministry of Women and Vulnerable Populations (MIMP); for which fourteen input variables were built, one relating to parenting style received, three family relationships, nine out of perceptions about gender roles and one referring to the geographical, seven output variables relate to the type of violence against women by their partner or ex-partner. For the design stage memory model and correlation perceptron network training apply the backpropagation algorithm, the activation function used in this case was sigmoidal (logistic), we calculated the mean square error to evaluate the error committed by the network in each pattern (observation), and finally generalized delta rule to change weights and thresholds. Two programs were developed in Matlab to implement the memory mapping model multilayer perceptron with one and two hidden layers. The model multilayer perceptron correlation memory with two hidden layers I provide better results, $80.34 \%$ of correct predictions, and shows that family violence and perceptions about gender roles are important factors in predicting the type of violence that women have ever joined or united by their partner, and reside in the sierra of Peru.
\end{abstract}


Keywords: Correlation memories, multilayer perceptron, predictions, violence against women

\section{Introducción}

Una de las formas más comunes de violencia contra la mujer es la causada por su marido (esposo) o pareja. Esto muestra una diferencia con la situación de los hombres, mucho más expuestos a sufrir agresiones de extraños o desconocidos, que de personas de su círculo íntimo. El hecho de que las mujeres a menudo tengan vínculos afectivos con el hombre que las maltrata $y$ dependan económicamente de él, ejerce gran influencia sobre la situación de maltrato y las estrategias para hacerle frente.

Violencia contra la mujer es - "cualquier acto o amenaza de violencia basada en género que tenga como consecuencia, o tenga posibilidades de tener como consecuencia, perjuicio y/o sufrimiento en la salud física, sexual o psicológica de la mujer. Consejo Económico y Social, ONU, 1992.

Estudios publicados por el Banco Interamericano de Desarrollo (BID), muestra que el crecimiento económico no resuelve los problemas de desigualdad de género y violencia física y sexual [1]. La violencia contra la mujer ocurre en todos los países, en todos los estratos sociales y está asociada al control sexual del hombre sobre la mujer y a los roles que cada sociedad asigna a las mujeres. Una de las grandes barreras para acabar con la violencia contra la mujer es la actitud de la sociedad y los estereotipos.

En Perú al igual que en muchos países latinoamericanos,la violencia contra la mujer es un problema ancestral, con explicaciones sociológicas y de género. En nuestro país los medios de comunicación contribuyen al asentamiento de estereotipos con producciones nacionales que ensalzan al macho, sacavueltero y pendejo y a la mujer sumisa, recatada, buena esposa que sufre en silencio.

Según datos de la Encuesta Demográfica de Salud Familiar 2011 [2], en el Perú el 65.6 por ciento de las mujeres unidas o alguna vez unidas manifestó que el esposo o compañero ejerció alguna forma de control sobre ellas; Según departamentos, los porcentajes más altos fueron en Apurímac y Pasco con 84.1 y 83.1 por ciento, respectivamente. El 11.3 por ciento de las mujeres unidas o alguna vez unidas manifestó que el esposo o compañero las amenazó con hacerles daño y el 21.3 por ciento con irse de la casa, quitarles los hijos(as) o la ayuda económica. Los departamentos donde las mujeres unidas o alguna vez unidas recibieron mayor porcentaje de amenazas "hacerle daño" son Apurímac y Cusco con 26.3 y 10.7 por ciento, respectivamente; San Martín, Apurímac, Junín, Cusco, son los departamentos con mayor porcentaje de amenazas "irse de la casa, quitarles los hijos(as), quitarles ayuda económica" con 30.9, $30.7,30.6$ y 30.2 por ciento, respectivamente. El 38.9 por ciento de las mujeres unidas o alguna vez unidas manifestaron que fueron víctimas de violencia física y sexual por parte de su esposo o compañero, como empujones, golpes, patadas, ataques o amenaza con cuchillo, pistola $u$ otra arma, así como tener relaciones sexuales sin su consentimiento o realizar actos sexuales que ellas no aprobaban; la mayor ocurrencia fue en el área urbana con 39.8 por ciento, en la región Selva con 42.8 por ciento y, en los departamentos de Junín y Cusco con 53.1 y 51.2 por ciento, respectivamente.

De enero a noviembre del 2012 los Centros de Emergencia Mujer (CEMs) del Programa Nacional contra la Violencia Familiar y Sexual del Ministerio de la Mujer y Poblaciones Vulnerables (MIMP) atendieron alrededor de 35 mil casos de violencia familiar y/o sexual.

La violencia contra la mujer es quizás la violación de derechos humanos más vergonzosa. $Y$ es quizás la más generalizada. No conoce ningún límite de geografía, cultura o riqueza. Siempre que siga, no podemos alegar estar logrando avances reales hacia la igualdad, el desarrollo y la paz. Secretaria General de las Naciones Unidas Kofi Annan, 1999.

La presente investigación pretende aborda esta situación aplicando métodos diferentes a los de la Estadística clásica; para encontrar el modelo que permita realizar predicciones acerca del tipo de violencia que sufren las mujeres de la sierra del Perú; y encontrar asociaciones importantes entre algunos estilos de crianza y la percepción sobre los roles de género, de la mujer unida o alguna vez unida con respecto a la violencia sistemática que padece por parte de su pareja o ex-pareja; aplicaremos el modelo perceptrón memoria de correlación multicapa que son modelos del Data Mining e Inteligencia Artificial (basado en el Aprendizaje Adaptativo) a la base de datos 
proporcionada por el Ministerio de la Mujer y Poblaciones Vulnerables (MIMP) [3].

Actualmente las técnicas basadas en el Aprendizaje Adaptativo causan gran impacto, debido a su extraordinaria aplicabilidad práctica.

Recientemente esta metodología ha captado la atención de los profesionales de la Estadística, los cuales comienzan a incorporar técnicas del Data Mining al conjunto de herramientas estadísticas orientadas a la clasificación de patrones, estimación y predicción.

\section{Metodología}

\subsection{Topología del Perceptrón Memoria de Correlación Multicapa}

Memoria de Correlación, define el mapa total entre la entrada y salida de la memoria asociativa binaria [4]:

$$
\hat{\mathbf{M}}=\sum_{i=1}^{k} \mathbf{y}_{i} \mathbf{x}_{i}^{T}=\mathbf{w}_{i}
$$

Perceptrón Multicapa, es una red neuronal de alimentación hacia adelante, compuesta por una capa de unidades de entrada, un número determinado de capas ocultas y una capa de unidades de salida. Las unidades de salida están conectadas solamente con las unidades de la última capa oculta, como se muestra en la figura 1.

La capa oculta realiza un procesamiento no lineal de las unidades de la capa de entrada, extrayendo las características más importantes.

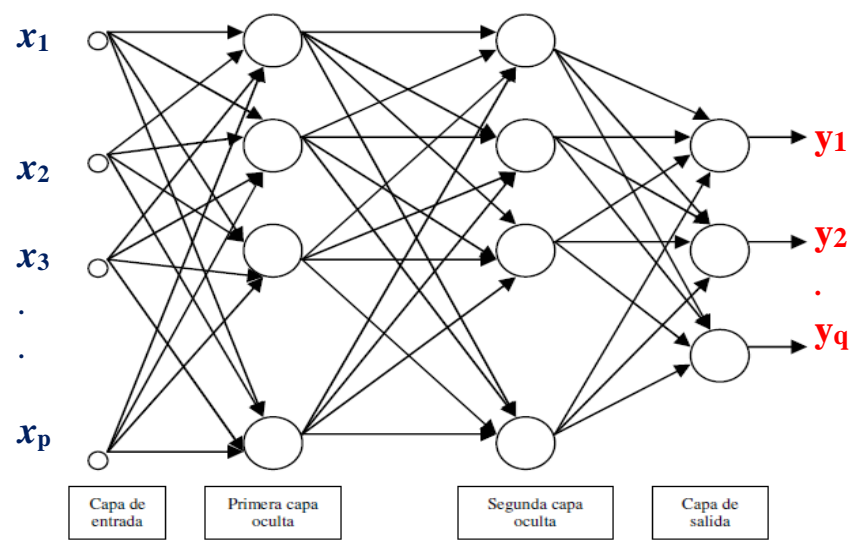

Figura 1: Topología de perceptrón multicapa

Perceptrón Memoria de Correlación Multicapa

$$
y_{i}=g_{1}\left(\sum_{j=1}^{M} w_{i j} s_{j}\right)=g_{1}\left(\sum_{j=1}^{M} w_{i j}\left(g_{2}\left(\sum_{r=1}^{L} t_{j r} x_{r}\right)\right)\right)
$$

$w_{i j}$ es el peso sináptico de la conexión entre la unidad de salida $i$ y la unidad de proceso $j$ de la capa oculta; $L$ es el número de unidades de proceso de la capa oculta; $g_{1}$ es la función de transferencia de las unidades de salida, para este caso utilizamos la función sigmoidal:

$$
g_{1}(n e t)=\frac{1}{1+e^{(-n e t)}}
$$

$t_{j r}$ es el peso sináptico que conecta la unidad de proceso $j$ de la capa oculta con el sensor de entrada $r$, y $g_{2}$ es la función de transferencia de las unidades de la capa oculta, que en este caso también es la función sigmoidal.

\subsection{Algoritmo de Aprendizaje (retropropagación)}

Objetivo ajustar los pesos sinápticos y sesgos para minimizar el error global o suma del error cuadrático (salida deseada menos salida, elevada al cuadrado).

$$
E=\sum_{k=1}^{p} \sum_{i=1}^{M}\left(z_{i}(k)-y_{i}(k)\right)^{2}
$$

Este método de aprendizaje supervisado, se realiza de forma continua, cambiando pesos y sesgos en la dirección contraria a la pendiente del error, a la que se denomina técnica del gradiente descendiente [5].

El funcionamiento del algoritmo de retropropagación del error es el siguiente: dado un patrón de entrada se aplica como estímulo a la primera capa de neuronas de la red, se va propagando por las siguientes capas hasta que llega a la capa de salida donde se compara la salida obtenida con la deseada. Luego se calcula el error global para cada neurona de salida, y este valor de error es transmitido hacia atrás, por todas las capas intermedias, y se van modificando sus pesos sinápticos según dicho error y los valores de las salidas de las unidades de proceso precedentes ponderados por sus pesos sinápticos.

La tasa de aprendizaje es $\alpha$ de modo que $0 \leq \alpha<1$, valores cercanos a uno pueden ocasionar inestabilidad en el funcionamiento de la red, para corregir este inconveniente se utiliza la regla delta generalizada:

$$
\Delta w_{i j}(k)=-\alpha \frac{\partial E}{\partial w_{i j}}=\alpha \delta_{i}^{2}(k) s_{j}(k)
$$


que se obtiene de la ecuación (2) y (4). Esta regla es de gran utilidad para redes neuronales de múltiples capas y funciones de transferencia no lineales y diferenciables.

\subsection{Predicción}

Como necesitamos encontrar un modelo de predicción del tipo de violencia, se determinará la función que mapea el conjunto de catorce variables de entrada en siete variables de salida.

Un ejemplo de modelo de predicción se muestra en la figura 2.

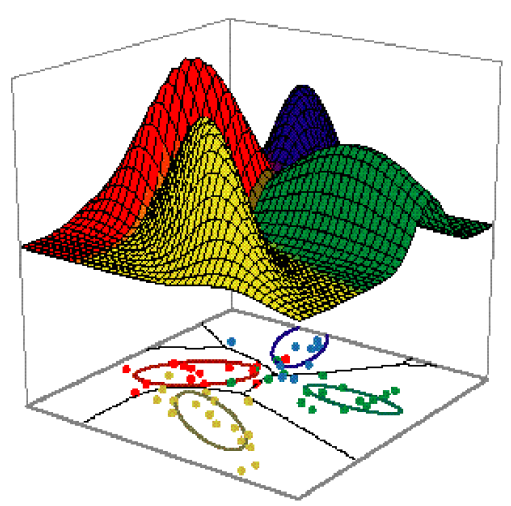

Figura 2: Modelo de predicción

\subsection{Validación}

El método de validación cruzada [6] consiste en dividir los datos de la muestra en dos partes; una se utiliza como conjunto de entrenamiento para determinar los parámetros del modelo, y la otra llamada conjunto de validación se utiliza para estimar el error de generalización mediante la tasa de predicciones incorrectas que proporciona el modelo, la que se obtiene con datos diferentes a los utilizados en el proceso de entrenamiento.

La muestra consta de 1126 mujeres mayores de 14 años unidas o alguna vez unidas residentes habituales en viviendas de las localidades circundantes de los CEM de la sierra del Perú.

Mediante el muestreo simple aleatorio seleccionamos aproximadamente el 90\% (1009 mujeres) para la etapa de aprendizaje de la red y el 10\% (117) restante como conjunto de validación.

\subsection{Variables}

Variables de entrada o explicativas:
$\mathbf{X}_{1}$ Cuando era niña sus padres la castigaban.

$\mathbf{X}_{2}$ Área de residencia.

$\mathbf{X}_{3}$ A quien le corresponde principalmente cocinar.

$\mathbf{X}_{4} \mathrm{~A}$ quien le corresponde principalmente lavar la ropa.

$\mathbf{X}_{5}$ A quien principalmente le corresponde decidir el momento de tener relaciones sexuales.

$\mathbf{X}_{6} \mathrm{~A}$ quien principalmente le corresponde mantener económicamente a la familia.

$\mathbf{X}_{\mathbf{7}} \mathrm{A}$ quien principalmente le corresponde encargarse de corregir a los hijos.

$\mathbf{X}_{8}$ A quien principalmente le corresponde decidir el uso de anticonceptivos.

$\mathbf{X}_{\mathbf{9}}$ A quien principalmente le corresponde llevar a los hijos para que los atiendan, cuando se enferman.

$\mathbf{X}_{10}$ A quien principalmente le corresponde ocuparse de los asuntos escolares de los hijos.

$\mathbf{X}_{11}$ A quien principalmente le corresponde hablar se sexualidad con los hijos.

$\mathbf{X}_{12}$ Ha sufrido agresión psicológica por algún miembro de su familia los últimos 12 meses.

$\mathbf{X}_{13}$ Ha sufrido agresión física por algún miembro de su familia los últimos 12 meses.

$\mathbf{X}_{14}$ Ha sufrido agresión sexual por algún miembro de su familia los últimos 12 meses.

Para $\mathbf{X}_{1}$, se consideró los siguientes castigos: cachetadas, reprimendas verbal o gritos, patadas, puntapiés, cocachos, golpes con látigos o correas, jalones de cabello 0 de oreja, dejándolos encerrado, echándoles o hundiéndolos en agua, entre otros.

Las preguntas $\mathbf{X}_{\mathbf{3}}$ a $\mathbf{X}_{\mathbf{1 1}}$ referidas a la percepción del rol de género, se codificaron de la siguiente forma:

$\begin{array}{rrl}\mathbf{X}_{\mathbf{i} 1} & \mathbf{X}_{\mathbf{i 2}} & \\ 0 & 1 & \text { Más los hombres. } \\ 1 & 0 & \text { Más las mujeres. } \\ 1 & 1 & \text { Ambos por igual. }\end{array}$

Las variables de salida (tipo de violencia ejercida por la pareja):

$Y_{1}$ Violencia psicológica por parte de la pareja.

$Y_{2}$ Violencia física por parte de la pareja.

$\mathbf{Y}_{3}$ Violencia sexual por parte de la pareja.

$\mathbf{Y}_{4}$ Violencia psicológica y física por parte de la pareja.

$\mathbf{Y}_{5}$ Violencia psicológica y sexual por parte de la pareja.

$\mathbf{Y}_{6}$ Violencia física y sexual por parte de la pareja.

$Y_{7}$ Violencia psicológica, física y sexual por parte de la pareja. 
En este caso el agresor es el esposo, conviviente, ex- esposo, ex - conviviente; en el caso de violencia sexual se considera además al enamorado/novio.

Las agresiones psicológicas consideradas fueron: insultos, burlas, desprecios, ignorada o rechazada, encerrada o recluida en su casa, impedida de ingresar a su vivienda, quitado o dañado sus pertenencias, prohibido estudiar o trabajar, impedida de ver o visitar a sus familiares o amistades, amenaza de daño físico, amenaza de muerte, otros.

Las agresiones físicas consideradas fueron: cachetadas, patadas, puñetazos, jalones de cabello o arrojándola contra la pared, arañazos, mordeduras; golpes con látigos, correas, soga, palos, leñas, bastones, piedras u otros objetos; ataques con armas punzocortantes como cuchillo, navaja, etc.; intento de ahorcamiento, asfixia 0 ahogamiento.

Las agresiones sexuales consideradas fueron: víctimas de manoseos, amenazas o acciones que las perjudicarían por no aceptar tener relaciones sexuales con alguna persona, alguien se ha masturbado o le ha mostrado sus genitales sin que ella lo haya deseado, ha sido víctima de penetración con dedos u objetos, ha sido víctima de intento de violación, practicado sexo oral a la fuerza.

\section{Resultados y discusión}

\section{Perfil de las entrevistadas:}

El $31.7 \%$ son mujeres jóvenes (entre 18 y 29 años), $61.8 \%$ adultas (entre 30 y 59 años).

$33 \%$ tienen nivel de instrucción primaria completa y $36.7 \%$ secundaria completa.

$51.4 \%$ no trabaja, $97.5 \%$ tiene hijos, $44.6 \%$ reside en el área rural; y con respecto a su estado civil $35.5 \%$ son casadas y $52.6 \%$ convivientes.

La figura 3 muestra que el $96 \%$ de las entrevistadas sufrieron castigos cuando eran niñas, por parte de sus padres.

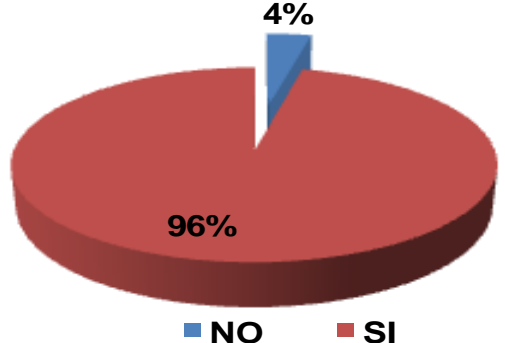

Figura 3: ¿Cuándo era niña sus padres la castigaban?

La figura 4 muestra que la mayoría (entre el $57.19 \%$ y $82.24 \%$ ) de las mujeres consideran que las labores del hogar, de relación de pareja y de crianza y cuidado de los hijos, les corresponde a ambos por igual.

La Tabla 1, muestra el tipo de violencia sufrido por las mujeres de la sierra del Perú por parte de su pareja o ex-pareja.

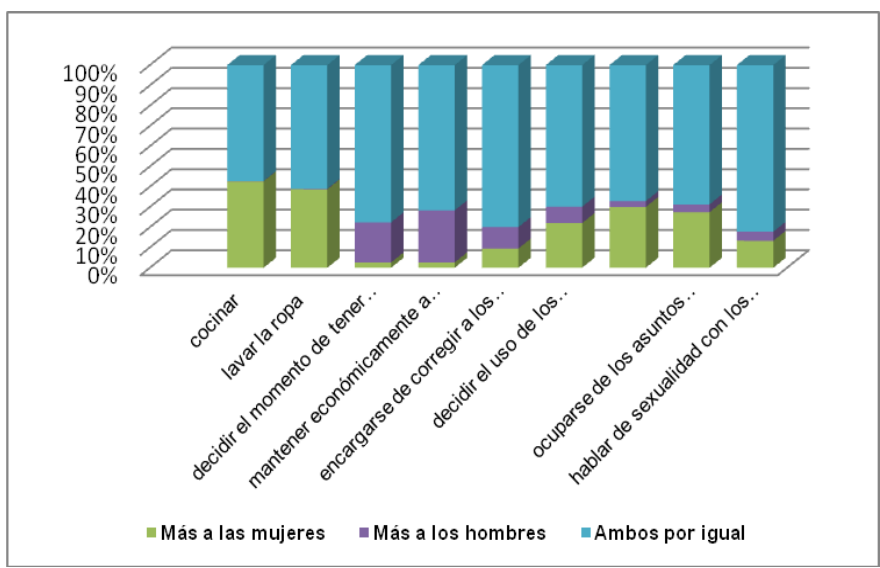

Figura 4: Percepción acerca de los roles de género

Tabla 1: Porcentaje de Mujeres unidas o alguna vez unidas agredidas por su pareja en la Sierra del Perú

\begin{tabular}{|l|c|c|}
\hline Tipo de violencia & Sí & No \\
\hline Psicológica & 43.3 & 56.7 \\
\hline Física & 27.2 & 72.9 \\
\hline Sexual & 21.2 & 78.8 \\
\hline Psicológica y física & 21.4 & 78.6 \\
\hline Psicológica y sexual & 15.2 & 84.8 \\
\hline Física y sexual & 11.9 & 88.1 \\
\hline Psicológica, física y sexual & 9.9 & 90.1 \\
\hline
\end{tabular}


Entrenamiento o aprendizaje del modelo perceptrón memoria de correlación multicapa.

Se elaboraron dos programas en Matlab R2010a, para el modelo de una y dos capas ocultas se trabajo en la fase de entrenamiento con 1009 casos con 14 variables de entrada y 7 de salida, los resultados se muestran en la Tabla 2.

Tabla 2: Resultados de la etapa de entrenamiento

\begin{tabular}{|l|c|c|}
\hline \multirow{2}{*}{ Descripción } & \multicolumn{2}{|c|}{ Modelo perceptrón memoria de correlación multicapa } \\
\cline { 2 - 3 } & $\begin{array}{c}\text { Tres capas, una de las } \\
\text { cuales es oculta }\end{array}$ & $\begin{array}{c}\text { Cuatro capas, dos de las cuales } \\
\text { son ocultas }\end{array}$ \\
\hline Tasa de aprendizaje & 0.2 & 0.2 \\
\hline Número de iteracciones & 505 & 324 \\
\hline Error global & 864.39 & 1043.7 \\
\hline
\end{tabular}

El modelo con una capa oculta muestra menor error global, pero el modelo de dos capas ocultas requiere menor tiempo computacional.

\section{Validación}

Con los modelos obtenidos en la etapa de entrenamiento, procedemos a validar los modelos para evaluar su capacidad de generalización, utilizaremos el segundo grupo de datos (117 casos). Los resultados se muestran en la siguiente tabla.

Tabla 3: Predicciones modelo perceptrón memoria de correlación multicapa

\begin{tabular}{|l|c|c|}
\hline \multirow{2}{*}{ Tipo de violencia } & \multicolumn{2}{|c|}{ Porcentaje de predicciones correctas con } \\
\cline { 2 - 3 } & Una capa oculta & Dos capas ocultas \\
\hline Psicológica & 36.75 & 36.75 \\
\hline Física & 55.56 & 78.63 \\
\hline Sexual & 70.09 & 85.47 \\
\hline Psicológica y física & 52.99 & 82.05 \\
\hline Psicológica y sexual & 56.41 & 90.59 \\
\hline Física y sexual & 61.54 & 94.02 \\
\hline Psicológica, física y sexual & 76.92 & 94.87 \\
\hline Total & $\mathbf{5 8 . 6}$ & $\mathbf{8 0 . 3 4}$ \\
\hline
\end{tabular}

Considerando los resultados de la Tabla 2 y 3 , para la misma tasa de aprendizaje $(\alpha=0.2)$ el modelo perceptrón memoria de correlación multicapa, dos de las cuales son ocultas, requiere menor tiempo computacional, muestra mayor porcentaje de predicciones totales correctas, así como también para cada una de las variables de salida.

Factores principales para la predicción de la violencia contra la mujer por su pareja

En general podemos concluir que todas las variables son importantes para determinar la predicción de la violencia contra la mujer, debido a que cuando se elimina una de las variables consideradas se incrementa el error global del modelo.

En la siguiente figura, la línea azul muestra el valor del error global y las líneas rojas indica en cuanto se incrementa el error global al eliminar una de las variables explicativas del modelo propuesto.

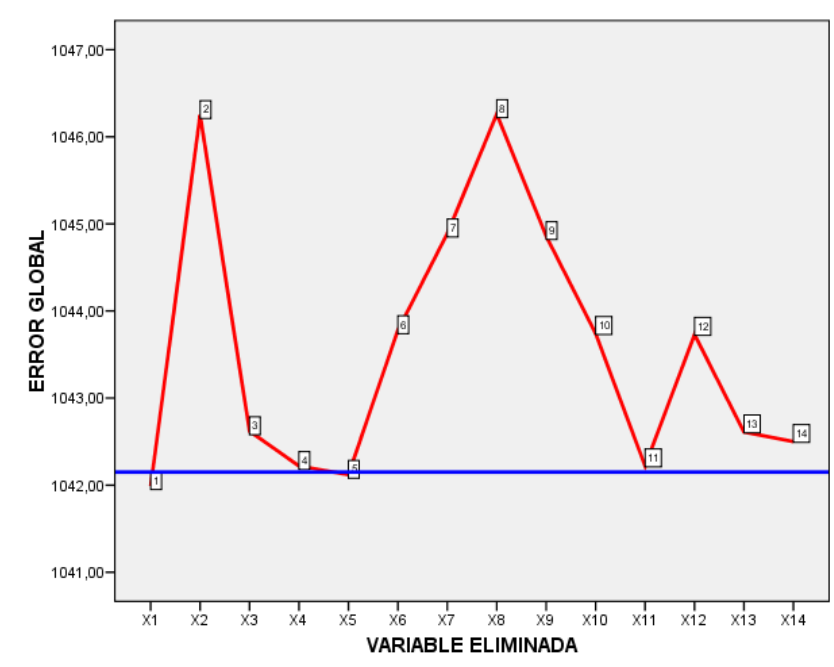

Figura 5: Error global eliminando una variable, del modelo perceptrón memoria de correlación cuatro capas dos de las cuales son ocultas.

\section{Conclusiones}

Las características más importantes de las mujeres unidas o alguna vez unidas de la sierra del Perú son las siguientes: la mayoría reside en las zonas urbanas de Ayacucho, Apurímac, Junín, Puno y Cusco; sus edades fluctúan entre 30 y 59 años, con estudios primarios y secundarios completos, no trabajan actualmente y tienen hijos(as); cuando niñas recibieron castigos psicológicos y físicos por parte de sus padres o de las personas que las cuidaban. La mayoría percibe que el hombre y a la mujer deben asumir por igual las responsabilidades en las tareas del hogar, cuidar y criar a los hijos(as), mantener económicamente a la familia, así como en aspectos relacionados con la vida sexual de la pareja.

Elegimos el modelo perceptrón memoria de correlación de cuatro capas dos de las cuales son ocultas, porque además de requerir menor tiempo computacional, proporciona el $80.34 \%$ de predicciones correctas. 
El modelo perceptrón memoria de correlación de cuatro capas dos de las cuales son ocultas, también nos proporciona las siguientes predicciones: $36.7 \%$ de las mujeres sufren violencia psicológica, $21.4 \%$ violencia física, $14.5 \%$ violencia sexual, $17.9 \%$ violencia psicológica y física, 9.4\% violencia psicológica y sexual, 5.9\% violencia física y sexual, $5.1 \%$ violencia psicológica, física y sexual, por parte de su pareja.

La violencia familiar y la percepción acerca del rol de género son factores importantes que están asociados con la violencia que sufre la mujer de la sierra del Perú por parte de su pareja.

\section{Agradecimientos}

Nuestro agradecimiento al Vicerrectorado de Investigación de la Universidad Nacional Mayor de San Marcos por el apoyo económico brindado para la realización de esta investigación.

También queremos agradecer a la Dra. Ana María Alejandra Mendieta Trefogli, Directora Ejecutiva del Programa Nacional contra la Violencia Familiar y Sexual del MIMP, por facilitarnos la base de datos con la que trabajamos los modelos propuestos en la presente investigación.

\section{Referencias}

[1] M.L. Biehl Violencia doméstica contra la mujer, Nota Técnica 7. División de Desarrollo Social del Departamento de Desarrollo Sostenible. Banco Interamericano de Desarrollo. Washington DC, (2002).

[2] Encuesta Demográfica y de Salud Familiar (ENDES) 2011. Instituto Nacional de Estadística e Informática (INEI). Lima-Perú, (Mayo 2012).

[3] Prevalencia, percepciones y necesidades de capacitación sobre violencia familiar y sexual. Ministerio de la Mujer y Desarrollo Social (MIMDES), Programa Nacional contra la Violencia Familiar y Sexual. Perú, (2009).

[4] D. Kustrin y J. Austin, Spiking Correlation Matrix Memory, Departament of Computer Science, (University of York, 1999).

[5] S.Haykin, Neural Networks a Comprehensive Foundation, Macmillan College Publishing Company, Inc. U.S.A. (1994).

E-mail: mepaunmsm@yahoo.es stonedblack@hotmail.com 